\title{
Arborescences
}

Revue d'études françaises

\section{Aborder les Relations jésuites de la Nouvelle France (1632-1672) Enjeux et perspectives}

\section{Adrien Paschoud}

Numéro 2, mai 2012

La littérature de voyage

URI : https://id.erudit.org/iderudit/1009268ar

DOI : https://doi.org/10.7202/1009268ar

Aller au sommaire du numéro

Éditeur(s)

Département d'études françaises, Université de Toronto

ISSN

1925-5357 (numérique)

Découvrir la revue

Citer cet article

Paschoud, A. (2012). Aborder les Relations jésuites de la Nouvelle France (1632-1672) : enjeux et perspectives. Arborescences, (2).

https://doi.org/10.7202/1009268ar
Résumé de l'article

Publiée chez l'éditeur catholique Sébastien Cramoisy entre 1632 et 1672, la collection des Relations forme une masse documentaire et scripturaire de premier plan pour qui s'intéresse à la colonisation des territoires nord-américains, mais également aux pratiques institutionnelles, doctrinales et savantes de la Compagnie de Jésus. Objet d'un intérêt renouvelé depuis les années 1980, cette publication, véritable vitrine de l'entreprise missionnaire des jésuites auprès d'un lectorat dévot et lettré, se prête à des approches croisées. Elle intéresse aussi bien les historiens et les ethnohistoriens que les spécialistes du littéraire et de l'esthétique de la réception ; elle touche également aux problématiques post-coloniales dans la mesure où elle interroge de plain-pied les rhétoriques de l'altérité (otherness) et les phénomènes de violence symbolique qui les accompagnent (une approche qu'un pan important de la critique, notamment dans le domaine de l'histoire culturelle, remet aujourd'hui en question pour privilégier une " anthropologie des zones de contact »). Cet article se propose donc de dresser un état des lieux bibliographique qui reflète plus largement la richesse d'un corpus qu'on ne saurait limiter à sa seule composante apologétique.
Tous droits réservés @ C Département d'études françaises, Université de Toronto, 2012
Ce document est protégé par la loi sur le droit d'auteur. L'utilisation des services d'Érudit (y compris la reproduction) est assujettie à sa politique d'utilisation que vous pouvez consulter en ligne.

https://apropos.erudit.org/fr/usagers/politique-dutilisation/ 


\title{
ABORDER LES RELATIONS JÉSUITES DE LA NOUVELLE FRANCE (1632-1672)
}

\author{
Enjeux et perspectives \\ Adrien Paschoud \\ Université de Lausanne
}

Depuis les années 1980, les travaux relatifs à la collection des Relations jésuites de la Nouvelle France, parue entre 1632 et $1672^{1}$, se sont multipliés, témoignant plus généralement du renouveau historiographique qui affecte aujourd'hui l'entreprise apostolique de la Compagnie de Jésus entre les $\mathrm{XVI}^{\mathrm{e}}$ et XVIII ${ }^{\mathrm{e}}$ siècles ${ }^{2}$. S'ils couvrent des champs de recherche d'une grande diversité (histoire de l'Église et des missions, histoire de la colonisation, ethno-histoire, New Indian History, études post-coloniales, histoire des pratiques littéraires, esthétique de la réception), ces travaux font part, à d'importantes exceptions près, d'une appréciation nuancée de l'apport de la culture jésuite au paysage intellectuel du XVII siècle. Ils se tiennent en effet à distance des a priori mutilants qui ont naguère prévalu au sein des milieux académiques, notamment dans le domaine de l'histoire littéraire française, durablement marqué par l'anti-jésuitisme pascalien et le radicalisme des Lumières. On ne peut que s'en réjouir au vu de la richesse exceptionnelle d'un corpus qui se prête de toute évidence à des approches croisées.

Il convient tout d'abord de rappeler que, pendant longtemps, la collection des Relations n'a été disponible que dans des rééditions laissées aux soins, et cela est logique, de la Compagnie de Jésus. Il en est ainsi des Monumenta Nova Franca ${ }^{3}$ procurées par Lucien Campeau (1967-), un tra-

\footnotetext{
${ }^{1}$ Composée de missives de longueur variable, accompagnée de divers documents, la collection des Relations de ce qui s'est passé en la Nouvelle France, parue chez le grand éditeur catholique Sébastien Cramoisy entre 1632 et 1672, obéit concurremment à plusieurs objectifs. S'adressant d'abord et avant tout un lectorat composé de dévots, de prêtres, de religieuses, elle poursuit - et cela est évident - des visées apologétiques : zèle et ferveur des missionnaires, conversions exemplaires, miracles, prodiges, intervention de la Providence, sanctification des martyrs, etc., sont autant d'appels à la foi. Mais les Relations sont également diffusées auprès d'un public plus large : lettrés, savants, administrateurs royaux, parlementaires provinciaux, financiers, marchands. Ces lecteurs y cherchent des informations relatives aux potentialités de la colonisation en Nouvelle France; ils répondent en cela aux sollicitations du pouvoir royal (celui-ci, par l'entremise de Richelieu, entend en effet favoriser l'accroissement démographique et économique de la Nouvelle France). Mais ce lectorat est également friand d'exotisme : les mœurs et coutumes «sauvages », ainsi que les données relatives à la faune, à la flore, aux configurations géographiques, au climat comblent ainsi un attrait pour les «singularités» du Nouveau Monde, et alimentent bien des spéculations sur l'homme primitif, la loi de nature, l'origine et le développement de la société, la croyance... Le succès de la collection est du reste considérable, du moins durant les vingt premières années de sa parution.

2 Voir l'état des lieux procuré par Fabre et Romano (1999 : 433-439).

${ }^{3}$ L'édition de Campeau s'inscrit plus largement dans la collection des « Monumenta Historica Sociatetis Iesu », constituée de recueils de sources et divisée en sous-séries par personnages ou par pays.
} 
vail certes solide sur le plan philologique, mais qui relève exclusivement d'une apologie pro domo (l'activité missionnaire s'y voit en effet magnifiée). S'illustrant par de remarquables travaux, l'école canadienne s'est attelée à dénoncer l'absence flagrante d'objectivité des Monumenta et les erreurs factuelles qui s'y trouvaient (Laflèche et Gagnon 1988-). Cette réévaluation était nécessaire tant les considérations de Campeau paraissent aujourd'hui bien naïves (les Hurons vivaient, écrit-il, «à l'âge de pierre » (1987: 356)) ; elle a corrélativement conduit les chercheurs canadiens à offrir à un public élargi plusieurs éditions des Relations en livre de poche. L'appareil critique qui accompagne les Relations des Pères Le Jeune et Brébeuf (1999 [1634] et 1996 [1635]) permet alors de mieux en mesurer les enjeux historiques, pré-ethnographiques et théologiques ${ }^{4}$. D’autres publications ont vu le jour, qu'il s'agisse de sources missionnaires concurrentes - notamment le remarquable récit du frère mineur Gabriel Sagard (1990, [1632]) - ou de textes connexes (récits des coureurs des bois, écrits de voyageurs libertins comme le Baron de Lahontan (1990, [1703])). Ces ouvrages jettent un éclairage transversal, souvent fort polémique, sur les Relations ${ }^{5}$. Il reste à souhaiter que d'autres Relations, voire l'ensemble du corpus, soient rendus accessibles (la seule édition existante, hormis celle de Campeau, est celle procurée naguère par Thwaites (1896-1901)) ${ }^{6}$.

S’il demeure à ce jour modeste dans son étendue, ce travail éditorial participe plus largement de la volonté de désenclaver la production missionnaire de son seul ancrage apologétique ce qui était indispensable au vu du soin extrême avec lequel la Compagnie de Jésus a forgé sa propre historiographie ${ }^{7}$ - et de l'inscrire dans des perspectives plus vastes, au sein desquelles la prise en compte des enjeux institutionnels est primordiale. L'étude des Relations, mises en parallèle avec d'autres corpus (missions de Chine ${ }^{8}$, du Japon, des Indes occidentales), est une démarche indispensable à qui veut saisir la nature même de l'épistolarité jésuite comme outil de propagande religieuse, mais aussi comme instrument de cohésion identitaire. L'analyse comparée montre que la Compagnie de Jésus a veillé en permanence à maintenir l'unité d'un corps apostolique dispersé, rappelant ainsi ses membres aux principes édictés dès la fondation de l'ordre par Ignace de Loyola (Giard 1991). Plus largement, et en raison même de son ouverture vers les cultures les plus lointaines, l'activité apostolique accompagne en pratique un idéal, né du combat contre la Réforme, celui d'une chrétienté universelle. Dans cette optique, les modèles de savoir convoqués

\footnotetext{
${ }^{4}$ Notons cependant que Theodore Besterman a édité les écrits de Jean de Brébeuf (1957).

${ }^{5}$ L'aventurier Nicolas Perrot stigmatise ainsi la propension jugée excessive des jésuites au martyre (1999 [1700] : 116 et 150).

${ }^{6}$ On signalera cependant deux éditions modernes: The Jesuit Relations. Natives and Missionaries in Seventeenth-Century North-America (2000) ; il s'agit en l'occurrence d'extraits des Relations à usage scolaire. Il existe également une réédition - aujourd'hui épuisée - en cinq volumes (1972).

${ }^{7}$ Outre les travaux de Campeau cités plus hauts, voir Bouchard (1958). À propos de la prise en charge par la Compagnie de Jésus de sa propre histoire, voir Fabre et Romano (1999 : 247-260).

${ }^{8}$ Voir à cet égard Li (2001).
} 
(libido sciendi d'inspiration aristotélico-thomiste, attrait pour le merveilleux, comparatisme religieux) et les principes missiologiques qui les accompagnent (notamment le principe de l'accomodatio) participent de cette volonté d'affirmer un universalisme de la foi devant toutes les formes d'hétérodoxie (réformée, mais aussi libertine - voir infra). Pourtant, comme le suggère Clossey (2008 ; voir également Tinguely 2008), ce resserrement institutionnel, mené par les autorités jésuites de Rome, ne va pas de soi. En effet, les missions jésuites - américaines, européennes, chinoises, etc. - ne se développent pas, comme on pourrait naïvement le penser, selon un schéma exclusivement centrifuge. De nombreux foyers de relations se sont constitués entre les missions, sans nécessairement que les autorités romaines exercent un contrôle absolu sur ceux-ci. La circulation des individus, des objets, des données pré-ethnographiques, etc., ouvre alors à un espace de connaissance inédit, affranchi momentanément d'une assise exclusivement européo-centrée - sur ce point, l'approche de Clossey ouvre à un domaine en pleine expansion, celui de la «globalisation» et de des connected histories, représenté notamment en France par les travaux de Gruzinski(2004).

La prise en compte des problématiques institutionnelles ne forme évidemment qu'un des volets de la démarche historienne. Cette dernière a aussi et surtout fait usage des Relations dans le cadre de l'étude de l'expansion coloniale et apostolique dans les territoires nord-américains (ce que l'on nomme parfois au XVII ${ }^{\mathrm{e}}$ siècle le « Pays d'en Haut», c'est-à-dire essentiellement la région du Saint-Laurent et des Grands Lacs). De ce point de vue, la masse documentaire formée par le corpus jésuite est d'une importance capitale ; elle est généralement mise en perspective avec d'autres sources en langues française ou anglaise (écrits missionnaires concurrents, documents émanant des autorités coloniales, récits de voyage, chroniques, mémoires, autobiographies spirituelles, correspondance, monographies savantes, etc.). La collection des Relations s'avère également précieuse lorsqu'il s'agit de mesurer les profonds bouleversements que les sociétés amérindiennes ont connus sous l'action conjuguée de l'expansion coloniale et de l'évangélisation (Delâge 1991 ; Deslandres 2003). Mais c’est plus particulièrement le savoir pré-ethnographique des jésuites $^{9}$ - avec lequel sans doute peu de publications du XVII ${ }^{\mathrm{e}}$ siècle sont à même de rivaliser hormis le récit de Sagard - qui a trouvé dans le domaine historiographique un champ d'application particulièrement fécond. Dérivant de l'ethno-histoire inaugurée par les travaux de Fenton (1957), la New Indian History, représentée notamment par Trigger (1976), Axtell (1985), White (1991) ou Richter (1992), se propose de donner une compréhension plus fine du tissu co-

\footnotetext{
${ }^{9}$ On y trouve décrites des catégories comme la guerre, les formes du pouvoir, la magie, la médecine, les fêtes, les cérémonies, le mariage, le corps, la chasse. Les récits cosmogoniques, les croyances, les rites funéraires, les formes d'adoration y occupent une place considérable : à titre d'exemple, les considérations de Brébeuf sur la conception de l'« âme » chez les Hurons relèvent d'une grande qualité d'observation (1996 [1635] : 114-121).
} 
lonial ; surtout, elle place au centre de ses préoccupations les modes de pensée et d'action des sociétés amérindiennes. Il ne s'agit pas de nier pas la destruction du monde sauvage sous l'action de la conquête, mais de circonscrire les phénomènes d'hybridation qui se sont - même subrepticement - produits. La colonisation devient ainsi le lieu d'infléchissements, de tentatives de persuasion, d'échanges réciproques, ce que White appelle un middle ground (1991 : x-xi). Du fait de leur réussite ou de leur échec, ces dynamiques interculturelles se révèlent dans tous les cas créatrices de sens. Plusieurs études de cas, fondées notamment sur les Relations, sont présentées : le commerce des fourrures, les mœurs sexuelles, les conversions, les alliances militaires, etc. Engagés dans une herméneutique mouvante des actions et des intentions, Indiens et Européens ont créé de manière impensée des métissages qui dépassent de loin l'acculturation forcée. Située à la croisée de plusieurs champs d'étude, l'analyse de White prend toutefois peu en compte les modèles de savoir et les instruments discursifs qui caractérisent les récits des voyageurs, notamment jésuites ${ }^{10}$.

Cette tâche est revenue tout d'abord au domaine des études post-coloniales, lesquelles ambitionnent de mesurer le corpus jésuite à l'aune d'un concept qui est devenu somme toute trop accueillant (mais sans doute l'a-t-il toujours été...) : l'altérité (Otherness). Il s'agit alors d'étudier les filtres - théologiques, épistémiques, rhétoriques - au travers desquels les sociétés amérindiennes sont représentées. Selon Sayre (1997), qui intégre les Relations dans un corpus plus large de textes français et anglais, l'évocation du monde sauvage oscille en permanence entre deux pôles antithétiques (negation et substitution). Empreint d'une conception rétrogressive de l'histoire, le regard occidental forge une axiologie foncièrement négative : les Indiens, quels qu'ils soient, n’ont nulle connaissance de Dieu, ni religion, ni morale, ni système politique, etc. Dans le même temps, cependant, et de manière apparemment contradictoire, les récits de voyage lisent le monde sauvage à la lumière du mythe de l'âge d'or (ou du primitivisme chrétien), faisant des territoires les plus lointains le lieu où s'incarne une humanité qui n'a pas été contaminée par les vices de la civilisation, voire par la corruption adamique. L'ouvrage de Blackburn (2004), fondé essentiellement sur les écrits du Père Le Jeune, s’inscrit pour sa part dans le prolongement des travaux de Foucault. Il s'agit de montrer en quoi l'écriture missionnaire constitue un instrument de domination symbolique, en raison même de ses objectifs de conversion. La restitution de la parole sauvage, très présente dans les Relations, est toute entière inféodée au filtre européo-centré des jésuites. Est ainsi niée la possibilité de représenter l'altérité sans exercer une contrainte symbolique dont la

\footnotetext{
${ }^{10}$ Gilles Havard a contesté la nature même du concept de middle ground, jugeant qu'il relevait d'une forme d'impossibilité, dans la mesure où les sources qui sont données n'émanent évidemment que des Européens (Havard 2003 : 15-16). Richard White (2011) a lui-même répondu à ces critiques dans la nouvelle édition qu'il a procurée de son ouvrage.
} 
finalité est dans le meilleur des cas inconsciente. Les Indiens hurons ne sont que des figures de papiers, des artefacts servant l'idéologie coloniale. Voisine des travaux célèbres de Said (1980) et de Pratt (1992) ${ }^{11}$, la thèse de Blackburn veut que les Pères jésuites, incapables de décentrer leur regard, aient été amenés à reproduire les schèmes de compréhension fournis par leur propre culture, niant de fait toute appréhension effective de l'altérité. La question de l'instrumentalistion de la parole sauvage renvoie plus largement à une problématique chère à Lévi-Strauss, qui veut que l'écriture ait marqué symboliquement la perte irrémédiable du monde sauvage ${ }^{12}$.

Dans le domaine des études littéraires françaises, certains travaux se sont réappropriés, à des degrés divers, cette violence idéologique. C'est ainsi que Laborie (2003: 18) défend l'idée selon laquelle toute lecture de nature anthropologique des sources jésuites est vouée à l'échec : ce serait se condamner, affirme-t-il, à de vagues commentaires qui ne rendraient absolument pas compte de la spécificité du corpus missionnaire. Dès lors, tout n'est que construction rhétorique au service d'une entreprise de renforcement institutionnel que la correspondance jésuite ne cesserait de mener, quelles que soient par ailleurs les cultures en présence :

Le paradigme indien des jésuites s'enracine dans les clichés véhiculés, depuis déjà cinquante ans, par tous les discours environnants sur l'Amérique. L'Indien, parce qu'il est l'élément libre, déplaçable et manipulable à volonté, va constituer une figure fondamentale dans laquelle toutes les autres vont venir se refléter. Comme forme à investir, il est à la fois une métonymie du pays et une métaphore du projet dans lequel tout scripteur se reconnait. Ainsi sa fonction topique permit d'en réduire totalement l'étrangeté, au point de faire de son remplissage l'objet réel des textes. En effet l'on peut affirmer que ce personnage n'est qu'une silhouette saisie parfois de dos, s'éloignant, parfois de profil comme un passant, parfois de face comme un miroir transparent. Ce traitement apparaît comme le dénominateur commun à tous les textes qui, au XVIe siècle, rendent compte du même sujet (Laborie $2003: 196)^{13}$.

Une lecture exclusivement «figurale» du corpus jésuite, sans être fausse, appelle cependant d'importantes nuances. Bien qu'elle ouvre à des pistes sans doute fécondes en ce qui concerne le lien entre référent et discours, elle se heurte néanmoins à une impasse inscrite dans son projet même. En frappant de suspicion toute tentative de restitution référentielle, elle rend impossible toute distinction entre fait et interprétation; prisonnière d'une forme d'essentialisme stratégique, elle s'interdit de fait d'historiciser les textes.

\footnotetext{
11 « The Imperial Eyes aims to be both a study in genre and a critique of ideology. Its predominant theme is how travel books by Europeans about non-Europeans parts of the world went (and go) about creating the 'domestic subject' of Euroimperialism; how they have engaged metropolitan reading publics with (or to) expansionist entreprises » (Pratt $1992: 4)$.

${ }^{12}$ On peut cependant proposer une lecture différente de la parole sauvage, telle qu'elle est rapportée dans les Relations : éludant la question de violence symbolique, Doiron (1991: 375-402) montre avec précision que les harangues sauvages rapportées par Paul Le Jeune et Jean de Brébeuf constituent des lieux communs rhétoriques issus de l'Antiquité, prompts à plaire et à instruire.

${ }^{13}$ Dans cet ouvrage consacré avant tout aux missions d'Amériques du Sud, Laborie consacre un bref chapitre aux Relations (171-177).
} 
Dans un article suggestif, Reichler s'est proposé d'écarter une interprétation strictement autotélique des Relations, la jugeant insuffisante (2002: 37-56). S’il y a lieu de combattre toute forme d'objectivisme naïf, le risque est de s'enfermer dans une conception unilatérale des sources jésuites, les conclusions étant déjà connues avant même d'aborder les textes. Comme le souligne Reichler, il est bien évidemment impossible de demander aux missionnaires jésuites du XVII ${ }^{\mathrm{e}}$ siècle de renoncer aux objectifs de conversion qui sont les leurs et qui conditionnent l'écriture des Relations, mais la possibilité d'une lecture somme toute moins réductrice est proposée, une lecture qui tienne compte de la confrontation entre les modèles préformés des jésuites et leur éventuel infléchissement au contact du monde amérindien. Inspiré par l'ouvrage de White cité précédemment, Reichler envisage alors la rencontre des cultures huronnes et occidentales non plus sous un angle idéologique, mais comme le lieu complexe d'interactions inscrites dans un discours adressé. Dans cette optique, les Relations font fusionner dans leur matérialité discursive deux sphères culturelles a priori incompatibles. Les Hurons, nous disent les Pères jésuites, ont un attrait particulier pour les antithèses : l'âme et le corps, le monde des hommes et le monde des esprits, l'élevé et l'obscène, le respect et la moquerie, le dédoublement et le travestissement. Si les jésuites ont été attentifs à ces phénomènes (du moins les ont-ils perçus commet tels), c'est parce qu'ils retrouvent une configuration théologique qui leur est propre, celle du goût pour la conciliation des contraires : le péché et la grâce, l'avilissement et la sublimation, la raison et la foi... En raison même de sa disposition holistique et de son attrait pour les singularités, l'esprit «baroque » des jésuites «a représenté une configuration favorable à la constitution d'un sol commun, une sorte de réussite momentanée et rare du contact interculturel » (Reichler 2002 : 54). L'intersection même partielle entre la spiritualité jésuite de la Contre-Réforme et les sociétés huronnes permet de situer l'activité apostolique dans une perspective non plus statique, laquelle reviendrait à mesurer les failles nécessairement ethno-centrées du savoir jésuite, mais dynamique. Cette «anthropologie dialogique » n'entre pas en contradiction avec une approche discursiviste, mais elle la complète, car elle lui est intrinsèquement liée. Frédéric Tinguely a cependant nuancé la portée de la thèse défendue par Reichler: «L'approche littéraire des problèmes d'interculturalité doit impérativement faire le départ entre la façon dont le texte représente un processus d'interaction ou d'hybridation (activité des truchements, activité de troc, évangélisation, amabassade, et ainsi de suite) et celle dont, le cas échéant, il participe d'un tel processus » (Tinguely 2011 : 334). S'il convient effectivement de tenir compte de cette distinction - ce que fait du reste Reichler -, la démarche de ce dernier rend justice à la double praxis de la démarche apostolique des jésuites : celleci s'exerce bien évidemment sur le terrain, étant consubstantielle à l'entreprise de conversion. Mais elle se voit redoublée dans le discours, car elle doit agir comme un puissant adjuvant de la 
foi auprès du lectorat dévot et lettré auquel s'adresse la collection. Mais c'est précisément - et paradoxalement - à l'intérieur de ce discours hautement codifié que peuvent se lire les traces d'une dynamique interculturelle, bien que celle-ci demeure somme toute parcellaire.

La démarche de Reichler rend indispensable la prise en compte de l'« horizon d'attente » des Relations, au sens que l'esthétique de la réception a donné à ce terme dans le champ des études littéraires, c'est-à-dire l'ensemble des conventions qui constituent les compétences d'un lectorat (considéré comme une entité collective) à un moment donné. D’autres travaux se sont proposés d'aborder les phénomènes de réécriture dont cette collection a été l'objet au sein de la culture imprimée de la Compagnie de Jésus: traités, sermons, périodiques, dialogues philosophiques, libelles et réponses, monographies savantes.... À titre d'exemple, la matière hagiographique des Relations, et plus particulièrement le martyre des Pères Lallement et Brébeuf (exécutés par les Iroquois en 1649), est réformée dans le sens d'une mystique de l'anéantissement de soi, de l'abandon et de la servitude heureuse dans la poésie spirituelle de Jean-Joseph Surin; on peut également songer au martyrologe richement illustré de Mathias Tanner (1630-1692) intitulé Societas Iesu usque ad sanguinis et vitce profusionem militans, in Europa, Africa, Asia, et America [...], paru à Prague en 1675 (Paschoud, 2011 : 123-135) ${ }^{14}$. Il s'agit dans tous les cas de montrer que les Relations sont infléchies au gré des intentions apologétiques et polémiques; les modifications apportées au texte-source varient considérablement et vont de la simple transcription à la reformulation complète, en passant par l'annotation, le commentaire, l'amplification. On peut mesurer de quelle manière la Compagnie de Jésus se sert d'un dispositif rhétorique éprouvé, l'aptum cicéronien, c'est-à-dire le style auquel l'orateur a recours selon les circonstances, les lieux ou le sujet, faisant ainsi de sa production imprimée une partition à plusieurs voix. En plein accord avec la tradition aristotélothomiste qui allie foi et raison, les imprimés jésuites du XVII ${ }^{\mathrm{e}}$ siècle sont tantôt dévolus à l'érudition et aux savoirs, tantôt à la puissance de la représentation et aux affects (Van Damme 2003 : 189-203). Plus largement, cette démarche met en avant la propension de la littérature missionnaire à agir puissamment - mais par des substrats de représentation diversifiés - sur le lecteur, participant plus largement de cette fusion entre théorhétorique et foi, la première étant bien évidemment subordonnée à la seconde (Fumaroli 1994).

Mais l'étude de la réception des Relations dans le paysage intellectuel du XVII siècle conduit également à s'interroger sur l'avers de la production apologétique, c'est-à-dire le « libertinage érudit » (quels que soient les problèmes de définition que pose ce terme), un champ de recherche qui a connu au cours de ces denières années une forte expansion (voir l'état des lieux procuré par

\footnotetext{
${ }^{14}$ Cette contribution se distingue des nombreux travaux qui portent sur la conception du martyre proprement dit dans les Relations (Laflèche, 1987 : 25-45 ; Berthiaume 1995 : 79-87 ; Sayre, 1997 : 248-304).
} 
Moreau 2007). Il s'agit alors de montrer de quelle manière les écrits missionnaires ont nourri les interprétations hétérodoxes du monde sauvage. Dans ses Quatre dialogues faits à l'imitation des Anciens, par Orasius Tubero (1632), François de La Mothe le Vayer convoque à plusieurs reprises les Relations des jésuites afin d'étayer l'idée selon laquelle la « vertu des païens » est un instinct naturel qui permet de distinguer hors de toute tutelle religieuse le bien du mal. Dans l'optique qui est la sienne, la description des croyances amérindiennes, et plus particulièrement l'évocation des mythes des origines, est susceptible de disqualifier l'hypothèse monogéniste, fondée sur les chronologies de l'Ancien Testament. Cela laisse alors à penser que les polythéismes, loin de dériver du monothéisme, l'ont au contraire façonné. On peut également songer à L'Autre Monde ou les États et empires du Soleil (posthume 1662) de Cyrano de Bergerac, une fiction sélénographique qui se plaît à construire une série de variations autour du récit fondateur de la Genèse. La Bible n'est qu'un récit parmi d'autres, invalidant toute notion d'autorité. Une prolifération d'énoncés caractérise alors l'origine du monde, laquelle se voit en quelque sorte saturée de variantes dont aucune ne saurait prévaloir ; à l’image de la démarche sceptique, le fonctionnement référentiel du langage est irrémédiablement entamé. Cyrano ne cite pas explicitement les Relations, mais on relèvera que les pages liminaires du roman situent l'action dans les territoires de la Nouvelle-France et mettent en scène le héros-narrateur, Dyrcona, et les jésuites. Le récit de Cyrano permet plus largement de se pencher sur la manière dont le romanesque parasite une sphère de discours qui lui est $a$ priori étrangère, faisant ainsi montre de sa remarquable capacité d'absorption et de réfraction. Aborder la pensée hétérodoxe engage en retour un faisceau de questionnements sur les stratégies éditoriales mises en œuvre par la Compagnie de Jésus pour contrer toute forme de libre-pensée. On peut alors mieux saisir l'inscription des Relations dans la production imprimée des jésuites, lorsqu'il s'agit de combattre les interprétations «sophistiques» de la croyance, celles qui réduisent l'ordre surnaturel à un simple échafaudage de mots (Paschoud 2010 : 101-112). Cette problématique engage les dispositifs énonciatifs des Relations, celles-ci devenant alors le lieu d'une double prédication: les récits de conversion, de miracles, ou de martyre s'adressent certes au lectorat dévot, mais ils sont également destinés à redresser les esprits égarés vers les vérités célestes ${ }^{15}$.

Ce bref parcours fait apparaître la remarquable vitalité des travaux liés aux Relations. Les orientations que nous avons décrites entrent plus largement en résonance avec les acquis les plus récents de la recherche qui ont visité à nouveaux frais, et avec pertinence, la culture jésuite du XVII ${ }^{\mathrm{e}}$ siècle : il suffit de songer aux travaux de Certeau (1982), Fabre (1992), Fumaroli (1994), Giard (1996), Jouhaud (2000), ou de Valentin (2001), pour ne se limiter qu’à la seule historiogra-

${ }^{15}$ C'est du reste un lieu commun de la littérature apologétique jésuite que de rapprocher les «Sauvages américains » des «libertins », et ce depuis au moins la Doctrine curieuse des beaux esprits de ce temps (1623) du prédicateur François Garasse. 
phie française. Dans la perspective qui est la nôtre, les études littéraires, ce corpus mériterait des prolongements pour ce qui a trait notamment à la question de l'exemplarité, de l'accréditation du témoignage ou encore du glissement figural qui s'opère entre le référent historique et la restitution stylistique de celui-ci. On ne peut donc qu'appeler de nos vœux une attention plus fine portée aux ressorts formels (et plus particulièrement à la tension entre codification générique et inventio), lesquels viendraient en quelque sorte seconder l'historicisation du corpus jésuite, dans le prolongement des orientations suggérées notamment par Réal Ouellet (2010).

\section{Références bibliographiques :}

Axtell, J. 1985. The Invasion within. The Contest of Cultures in Colonial North America. New York and London : Oxford University Press.

Berthiaume, P. 1995. «Les Relations des jésuites : nouvel avatar de la Légende dorée ». Dans Figures de l'Indien, sous la direction de G. Thérien. Montréal : L'Hexagone : 79-87.

Blackburn, C. 2004. Harvest of Souls. The Jesuit Missions and Colonialism in North America (1632-1650). Montréal : McGill-Queen's University Press.

Bouchard, J. 1958. Le R.P. Paul Le Jeune, s.j. et la fondation des missions des Jésuites en Nouvelle-France, 1632-1642. Étude des méthodes missionnaires. Rome : Excerpta ex dissertatione ad lauream in Facultate Missiologica Pontificæ Universitatis Gregorianæ.

Brébeuf, J. de. 1957. Les Relations de ce qui s'est passé au Pays des Hurons (1635-1648) / Saint Jean de Brébeuf, édition préparée et commentée par T. Besterman, Genève : Droz.

- 1996. Écrits en Huronie. Édition préparée et commentée par G. Thérien, Montréal: Bibliothèque Québécoise [1635].

Campeau, L. 1967-. Monumenta Novae Franciae. Rome : Institutum historicum Societas Iesu. 1987. La Mission jésuite chę les Hurons (1634-1650). Montréal : Bellarmin.

Certeau, M. de. 1982. La Fable mystique (XVIe-XVII siècle). Paris : Gallimard.

Clossey, L. 2008. Salvation and Globalization in the Early Jesuit Missions. Cambridge : Cambridge University Press.

Delâge, D. 1991. Le Pays renversé. Amérindiens et Européens en Amérique du Nord-Est (1600-1664). Montréal : Boréal.

Deslandres, D. 2003. Croire et faire croire. Les missions françaises au XVII siècle. Paris : Fayard.

Doiron, N. 1991. «Rhétorique jésuite de l'éloquence sauvage au XVII ${ }^{\mathrm{e}}$ siècle. Les Relations de Paul Le Jeune (1632-1642) ». XVII siècle : 375-402.

Fabre P.-A. et A. Romano. 1999. Revue de synthèse 2-3 : 247-260.

Fabre, P.-A. 1992. Ignace de Loyola. Le lieu de l'image. Paris : Vrin.

Fenton, W. 1957. American Indian and White Relations to 1830. Needs and Opportunities for Study. Chapel Hill : North California University Press.

Fumaroli, M. 1994. L'École du silence. Le sentiment des images au XVII siècle. Paris : Flammarion. 
Giard, L., dir. 1991. «Introduction aux Lettres et instructions ». Dans Ignace de Loyola, Écrits, sous la direction de M. Giuliani, Paris : Desclée. 1996. Les Jésuites à l'âge baroque (1540-1640). Grenoble : Jérôme Million.

Greer A. 2000. The Jesuit Relations. Natives and Missionaries in Seventeenth-Century North-America. Boston/New-York : Bedford/St-Martins.

Gruzinski, S. 2004. Les Quatre parties du monde. Histoire d'une mondialisation. Paris : La Martinière.

Havard, G. 2003. Empire et métissage. Indiens et Français dans le Pays d'en Haut. Montréal et Paris : Septentrion et Presses Universitaires de la Sorbonne-Nouvelle.

Jouhaud, C. 2000. Les Pouvoirs de la littérature : histoire d'un paradoxe. Paris : Gallimard.

Laborie, J.-C. 2003. Mangeurs d'homme et mangeurs d'âme. Une correspondance missionnaire au XVIe siècle: la lettre jésuite du Brésil (1549-1568), Paris : Honoré Champion.

Laflèche, G. 1987. «Les jésuites de la Nouvelle France et le mythe de leurs martyrs ». Dans Les Jésuites parmi les hommes aux XVI et XVII siècles, sous la direction de G. Demerson et alii. Clermont-Ferrand : Presses Universitaires de Clermont-Ferrand : 25-45.

Laflèche, G. et Gagnon, F.-M. 1988-. Les Saint martyrs canadiens, Laval et Québec : Singulier.

Lahontan, L. A. de Lom d'Arce de, Euvres complètes. 1990. Édition préparée et commentée par R. Ouellet et A. Beaulieu. Montréal : Presses de l’Université de Montréal [1703].

Le Jeune, P. 1999. Un Français au pays des «bestes sauvages ». Édition préparée et commentée par A. Beaulieu. Montréal : Comeau et Nadeau [1634].

Li, S. 2001. Stratégies missionnaires des jésuites français en Nouvelle-France et en Chine au XVII siècle. Saint Nicolas / Paris : Presses de l'Université Laval / L'Harmattan.

Moreau, I. 2007. "Guérir du sot». Les stratégies d'écriture des libertins à l'âge classique. Paris : Champion.

Ouellet, I. 2010. La Relation de voyage en Amérique (XVI'-XVIII siècle). Québec: Presses de l’Université Laval.

Paschoud, A. 2010. «Les cosmogonies amérindiennes au miroir du discours missionnaire jésuite : l'exemple des Relations en Nouvelle-France (1632-1672)». Dans Nature et surnaturel. Philosophies de la nature et métaphysique aux XVI-XVIII siècles, sous la direction de V. Alexandrescu et R. Theis. Hildesheim : Georg Olms Verlag : 101-112.

. 2011. «Réécritures hagiographiques : Jean-Joseph Surin et Mathias Tanner lecteurs des Relations jésuites de la Nouvelle France (1632-1672)». Dans De l'Orient à la Huronie. Écritures missionnaires et littérature d'édification aux XVT et XVII siècles, sous la direction de G. Poirier. Laval : Presses Universitaires de Laval : 123-135.

Pioffet, M.-Ch. 1997. La Tentation de l'épopée dans les «Relations» des jésuites. Sillery : Éd. du Septentrion.

Pratt, M.-L. 1992. Imperial Eyes. Travel Writing and Transculturation. London and New-York: Routledge.

Reichler, C. 2003. «Littérature et anthropologie. De la représentation à l'interaction dans une Relation de la Nouvelle France au XVII ${ }^{\mathrm{e}}$ siècle ». L'Homme 164 : 37-56.

Relations des jésuites. 1972. Montréal : Éditions du jour (1611-1672).

Richter, D. 1993. «Whose Indian history?». The William and Mary Quarterly 50 : 379-393.

1992. The Ordeal of the Long-House. The Peoples of the Iroquois in the Era of European Colonization. Chapel Hill : University of North Carolina Press. 
Sagard, G. 1990. Le Grand voyage au pays des Hurons. Édition préparée et commentée par R. Ouellet et J. Warwick, Montréal : Bibliothèque Québécoise [1632].

Said, E. 1978. Orientalism. London : Routledge.

Sayre, G. M. 1997. Les Sauvages Américains. Representations of Native Americans in French and English Colonial Literature. Chapel Hill : North Carolina University Press.

Thwaites, R G. 1896-1901. The Jesuit Relations and Allied documents. Travels and Explorations of the Jesuit Missionaries in New France (1610-1791). Cleveland : Burrows.

Tinguely, F. 2008. «Le monde multipolaire des missionnaires jésuites». Dans La Renaissance décentrée, sous la direction de F. Tinguely, Genève : Droz : 62-72.

—. 2011. «État présent. Écritures du voyage à la Renaissance ». French Studies 44 : 329-335.

Trigger, B. 1976. The Children of Aataentsic. Toronto : McGill University Press.

Valentin, J.-M. 2001. Les Jésuites et le théatre (1554-1680). Contribution à l'bistoire culturelle du monde catholique dans le Saint-Empire romain germanique. Paris : Desjonquères.

Van Damme, S. 2003. «Les martyrs jésuites et la culture imprimée à Lyon au XVII siècle ». Revue des Sciences Humaines 269 : 189-203.

White, R. 2011. The Middle Ground. Indians, Empires and Republics in the Great Lakes Region (16501815). Cambridge : Cambridge University Press [1991] 